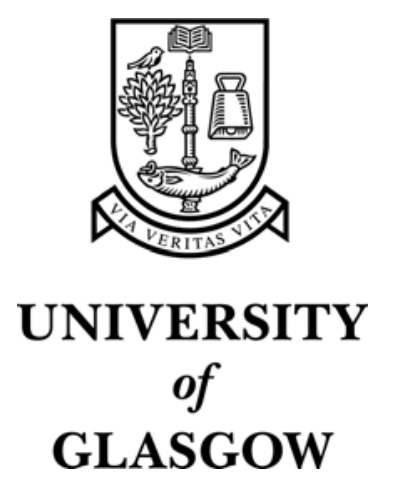

Yu, W. and Brewster, S.A. (2002) Multimodal virtual reality versus printed medium in visualization for blind people. In, Jacko, J.A., Eds. 5th International ACM Conference on Assistive Technologies, 8-10 July 2002, pages pp. 55-64, Edinburgh, Scotland.

http://eprints.gla.ac.uk/3229/ 


\title{
Multimodal Virtual Reality Versus Printed Medium in Visualization for Blind People
}

\author{
Wai Yu, Stephen Brewster \\ Glasgow Interactive Systems Group \\ Department of Computing Science \\ University of Glasgow, Glasgow, G12 8QQ, U. K. \\ +441413304966 \\ \{rayu, stephen\}@dcs.gla.ac.uk \\ http://www.multivis.org
}

\begin{abstract}
In this paper, we describe a study comparing the strengths of a multimodal Virtual Reality (VR) interface against traditional tactile diagrams in conveying information to visually impaired and blind people. The multimodal VR interface consists of a force feedback device (SensAble PHANToM), synthesized speech and non-speech audio. Potential advantages of the VR technology are well known however its real usability in comparison with the conventional paper-based medium is seldom investigated. We have addressed this issue in our evaluation. The experimental results show benefits from using the multimodal approach in terms of more accurate information about the graphs obtained by users.
\end{abstract}

\section{Keywords}

haptics, assistive technology, multimodal interface, human computer interaction, virtual reality

\section{INTRODUCTION}

The aim of this work is to address problems of blind people's access to graphical information particularly in visualizations such as line graphs, bar charts and pie charts. These are very commonly used to present data in an easyto-interpret way. Trends and distributions of data can be illustrated more effectively on a graph than on a table filled with raw data. Therefore, graphs are frequently used in economics, mathematics and other scientific subjects. Unfortunately, this kind of data visualization technique is not so useful to blind people. Being unable to access graphical information easily is a major obstacle to blind people in pursuing a scientific study and career. [1]

Traditionally, to make graphs accessible to blind people visual to tactile conversion is required to compensate for the loss of sight using special paper. The contents of a graph are raised to a different height than the background so that they can be discriminated by the cutaneous sense on people's fingers. Tactile diagrams exist in many forms and they mainly differ in the construction techniques and the materials used [2]. The most common type of tactile diagrams is a raised graph on swell paper. They are relatively easy to make and cost less when compared to other forms. Tactile diagrams provide blind people with possibilities to access graphical information however they are not very effective due to the characteristics of haptics and the limitations of the representation medium. Haptics is a much slower communication channel than vision. Perceiving information through the haptic channel is thus less efficient. Moreover, production of tactile diagrams is quite tedious and some training for blind people is required for successful use.

Attempts have been made to improve the representation of existing tactile diagrams by adding audio feedback. As a result audio tablets have been developed to use in conjunction with tactile diagrams [3,4]. A tactile diagram is placed on top of a touch sensitive tablet which stores the content information about the diagram. Audio information can then be given when users touch the object of interest on the diagram. Therefore, more information can be conveyed to users through the additional channel. Blind people can have a better idea about the displaying graph than before. In spite of this, the audio tablets still rely on the haptic representation of tactile diagrams and thus suffer from the same limitations as tactile diagrams. In addition, the device has to be programmed before use, as the information about the graph has to be stored first. Any changes to the graph will require modifications to the program. Therefore, these devices are still not the best solution to solve blind people's accessibility problems.

We are working on improving blind people's access to graphs and tables. Our research is based on the multimodal approach (using multiple sensory modalities) which uses haptics combined with audio and synthesized speech. Force feedback devices such as the SensAble PHANToM, 
Pantograph, Logitech WingMan Force Feedback Mouse, have been proven to be useful in exploring $3 \mathrm{D}$ objects, the graphic user interface and scientific simulations [5-7]. In our work, force feedback devices are used to provide blind people with the sense of touch on the virtual graphs rendered by the computer. The use of these devices gives us possibilities to overcome some shortcomings of tactile diagrams. Combining haptic and audio modalities enables us to present information according to the strengths of each modality. Our previous experiments have shown that the multimodal interface is feasible and effective to relay graph information to blind people [8]. In this paper, we introduce our evaluation of the multimodal interface in comparison with tactile diagrams in presenting graph information to blind people. Advantages and limitations of these two representation mediums will be given and the findings of the evaluation will be discussed.

\section{COMPARISON OF THE MULTIMODAL VR SYSTEM AND TACTILE DIAGRAMS}

In this study, the usability of the multimodal VR system and tactile diagrams is the major issue of investigation. The characteristics of these two media have a significant influence on their performance. Table 1 lists their characteristics according to various aspects.

Table 1. Characteristics of tactile diagrams and the mulitmodal VR system.

\begin{tabular}{|l|l|l|}
\hline Category & Tactile Diagram & VR System \\
\hline Cost & Low & Varies \\
\hline Production time & Long & Short \\
\hline Easy to change & No & Yes \\
\hline Dynamic & No & Yes \\
\hline Durability & Low & High \\
\hline Familiarity & High & Low \\
\hline Dimension & Two & Three or more \\
\hline Modality & Single & Multiple \\
\hline Haptic sense & Cutaneous & Kinesthetic \\
\hline Bandwidth & High & Low \\
\hline
\end{tabular}

Based on their characteristics, advantages and disadvantages of these two media in presenting graphical information can be drawn. The characteristics are related to different aspects which can be classified into three categories: production, interpretation and cognition, and practical usage.

In general, the cost of producing a tactile diagram is very low however that does not take into account of the initial investment of a computer, a printer, a photocopier and an embosser. The production process can be quite time consuming as it involves several steps. First of all, the graph has to be printed and then photocopied onto a swell paper. Afterwards, the paper has to be heat treated in order to raise the darkened parts. These tasks usually have to be carried out by a sighted person as some conversions are needed to make sure that the graph contents can be represented in the tactile form. These conversions usually involve removal of redundant information, i.e. gridlines, translating texts into Braille, and replacing colours with texture patterns, etc. After the graphs have been raised, some verification or proof reading is usually carried out to check any error or ambiguity of the raised information. Therefore, the production time could be affected by the amount of conversions needed and the skill of the person who carries out the tasks. It is not easy to make changes to tactile diagrams. Reheating the swell paper could overcook the already raised parts reducing the quality. Tactile diagrams are not very durable. After frequent use, the raised objects can be depressed or worn out. Moreover, there is a hygienic issue, after being used by many users, tactile diagrams often become very dirty and they cannot be cleaned simply by using a wet cloth.

On the other hand, tactile diagrams are more familiar to blind people who can use all their finger tips to extract information. They mainly use cutaneous sense to detect raised objects while their kinesthetic and proprioceptive senses are used to orientate themselves on the graphs. Both hands can be used on the tactile diagrams so that more information can be picked up simultaneously. Information is presented on a 2D surface and on most tactile diagrams all objects are raised to the same height. As a result, the vertical spatial resolution is very limited and the variety of information can be displayed on this $2.5 \mathrm{D}$ medium is restricted.

The cost of VR systems varies and depends on the force feedback device used. The SensAble PHANToM is extremely expensive (over \$10, 000 US for a desktop version) but the Logitech WingMan Force Feedback mouse is within the reach of most people (about $\$ 60$ US). As all graphs produced by the system are virtual, there are not extra production costs. Moreover, the time taken to produce a virtual graph is quite short as long as the rendering technique and the power of the computer is efficient and sufficient. Making changes on a virtual graph is relatively easy and only requires re-rendering of the graph. The quality of virtual graphs does not degrade over time so they are more robust.

However, virtual graphs are a novel medium unheard of by most blind people. Therefore it may take them some time to familiarize themselves with the new interface. The limitations of the force feedback devices also hinder users' exploration on the graphs. The one single point of contact greatly reduces the information which can be transmitted to the user at a given time. Moreover, only kinesthetic feedback is available so that users will not be able to use their full haptic sense. As the result, to explore a virtual 
graph could be more time consuming. Nevertheless, additional modalities can be incorporated in the VR system in order to compensate the limitations of force feedback devices. Both speech and non-speech audio can be an effective tool for users in accessing information which cannot be presented easily by haptics [8]. Furthermore, some force feedback devices can model 3D objects and thus the spatial resolution is much higher.

Based on these characteristics, both tactile diagrams and the multimodal VR system have their own advantages and limitations in presenting graphical information to blind people. It is unknown how they would compare in a real world application. Whether their strengths would overcome their limitations or the other way round. How well can users use these two media in performing tasks and perceiving information? In order to answer these questions we have conducted an experiment to test the real usability of the multimodal VR system in comparison with traditional tactile diagrams. In the experiment, we have tested how well people can use bar charts presented on these two media to answer a set of questions regarding the graph contents. In the following sections we will introduce the implementation of the mulimodal VR system and then the details of the evaluation.

\section{MULTIMODAL VR SYSTEM}

The multimodal VR system consists of two basic components: an IBM compatible PC and a force feedback device - SensAble PHANToM (Figure 1). An alternative force feedback device can be used such as a Logitech WingMan Force Feedback Mouse. The reason that we use SensAble PHANToM is because of its high fidelity of force feedback [8]. Various types of graphs and tables have been implemented on this system. The type of graph used for the evaluation is the bar chart.

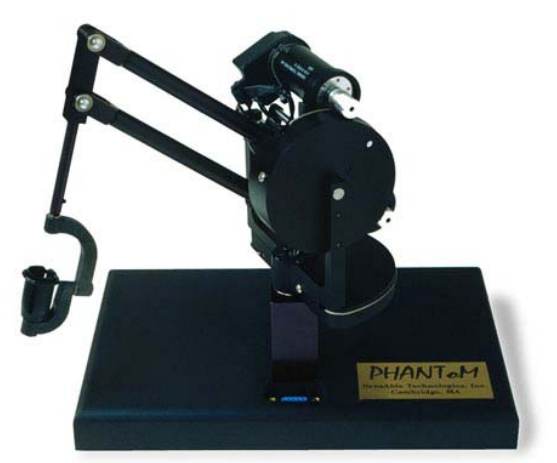

Figure 1. PHANToM from SensAble Technologies Inc.

Bar charts are a common type of graph used to present data trends and differences between data variables. Their simplicity and regularity in the graphical arrangement make it a suitable candidate for the evaluation. Furthermore, we have used bar charts in our previous experiments which investigated the strengths and weaknesses of two force feedback devices: the SensAble PHANToM and the Logitech WingMan Force Feedback Mouse [8]. In that experiment, the SensAble PHANToM showed clear advantages over the WingMan mouse in relaying information to users through haptic channel. However, when audio was introduced into the system, the difference between two devices became insignificant. Based on the experiment findings and the comments given by the participants, improvements have been made to the existing mulitimodal bar charts. Therefore, they are fully developed and suitable for this comparison study on the difference between the virtual graphs and tactile graphs.

The bar charts include haptics, synthesized speech and nonspeech audio. The haptics provides a model of the virtual bar chart which can be interacted with using the SensAble PHANToM. The synthesized speech presents precise information about the data variables on the bar chart. The non-speech audio consists of MIDI notes which provide a quick indication of the data trend as the pitch of the sound is mapped to the bar height.

The system has been developed to generate bar charts automatically based on raw data. It reads data sets stored in text files and renders them into virtual bar charts. The rendering process includes several steps such as scaling data, building a haptic model and mapping MIDI notes.

\section{Data Scaling}

The text files usually contain a series of data which is an arbitrary size. Each data variable is represented by a bar on the graph. In order to fit all the bars into the display window, scaling is needed. Moreover, cautions have to be taken that only the appropriate portion of the bars is rendered. This is because some data sets contain very similar values which would look identical if they are directly rendered into bars (Figure 2a). Therefore, a selective scaling is needed so that the upper part of the bars is magnified and displayed (Figure 2b).

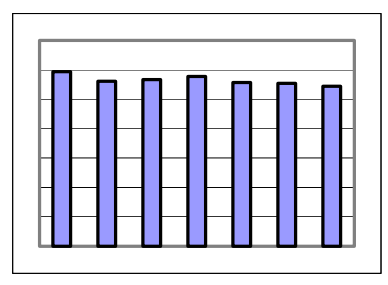

(a)

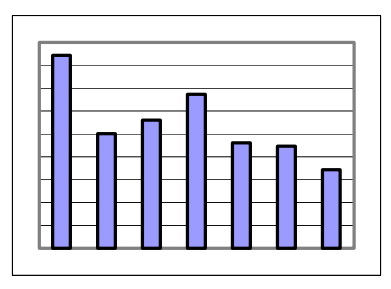

(b)
Figure 2. (a) original graph, (b) scaled graph.

To determine whether or not the selective scaling is needed, a condition checking on the data is performed. First of all, a simple criterion is set to check whether the data set needs to be modified. The maximum and minimum data variables will be determined. The ratio of the 
difference between these two variables and the minimum data variable will be compared with a predefined threshold value. If the ratio is lower than the threshold value then the selective scaling is required.

To achieve the selective scaling, some modification to the data set will be performed. A new baseline which is a portion of the minimum variable will be calculated. The difference between each variable and the baseline will be amplified by a predefined scaling factor and the result will be used as the height of the bar for rendering. Therefore, variations between each data variable will be easier to distinguish. For the data sets which do not meet the criterion of the selective scaling, the original value of each data variable will be scaled and used as the bar height.

\section{Haptic Bar Chart Modeling}

The haptic bar chart modeling is based on the general arrangement of its graphics counterpart therefore blind people can have the same impression of bar charts as sighted people. However, simplifications have been made on the haptic representation so that items like gridlines, legends, labels are not rendered. This is because their functions in haptics are not as useful as in visual and can be replaced by the synthesized speech.

The main items on the haptic bar chart are X \& Y axes and bars. The haptic rendering of these items is different. Both concave and convex shapes are used. The axes are modeled as cylinders which are raised from the background whereas bars are concave and engraved (Figure 3). The distinctive feature is aimed at showing users about the difference between these two items. Putting bars in the concave shape is due to the findings of our previous experiments in which users found it hard to keep the pointer on the raised curves securely [9]. Concave shape is thus used to solve this problem.

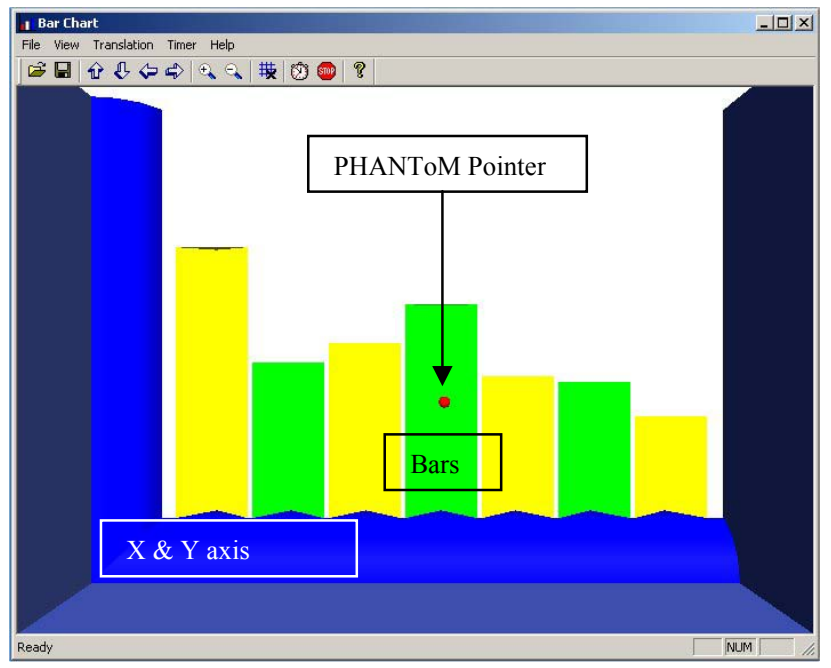

Figure 3. A snapshot of the haptic bar chart.
Axes are stationary objects while bars are changed according to the data set being rendered. Polygons are used to construct the bars which are placed closely together. This arrangement of bars is done according to the design guidelines of tactile diagrams $[2,10]$. There is a small gap between the $\mathrm{Y}$ axis and the first bar on the left. The results from the data scaling stage are used in the haptic bar rendering process. No haptic texture is created on any objects so that they feel smooth.

\section{Audio Implementation}

The audio features on the bar charts are designed to provide additional information to users. Besides getting haptic feedback, users are presented with speech and nonspeech sound which contains information about the data. Users deal with the sounds in an interactive fashion. The amount of information, type of sound and its occurrence are under users' control. Users interact with the sound through the PHANToM device. The position of contact point and the switch state on the PHANToM stylus determine the type of information being presented. The synthesized speech is used to inform users about precise value of each bar on the graph. The non-speech sound consists of different MIDI notes whose pitch is mapped to the height of the bar. Therefore, an abstract information about the data is presented, but much more rapidly.

The speech on the bar charts is rendered through the use of Microsoft Speech SDK. It is activated by pressing the switch on the PHANToM stylus when the pointer is in contact with a bar. There are two speech modes and their occurrence is determined by the number of clicks on the switch. On the single click, a bar's height on the graph will be read out. The bars' height is determined in the data scaling stage. On the double click of the switch, the exact value of a bar, which is un-scaled, will be given. Thus, users have the flexibility to choose which type of data that they are interested in. Single click provides easy-tomemorize information whereas double click gives precise details.

MIDI notes are intended to give users a quick indication of the bar height. The height of a bar is mapped proportionally to the pitch of a MIDI note. A tall bar will give a high pitch and vice versa. The sound is triggered by the contact between the PHANToM pointer and a bar. Therefore, by moving the pointer across all the bars on the graph, a series of MIDI notes will be played and the ups and downs of the pitch will indicate the highs and lows of the bars. A quick overview of the data trend will be perceived by users.

The MIDI synthesizer on the computer sound card is used to generate the sound. The piano timbre is chosen to be the musical instrument because of its support of broader range of notes. However, care has to be taken not to use the full range of 128 MIDI notes because many sound cards do not have the full support of all MIDI notes. At two extremes: very low and high, notes are often inaudible. Therefore, 
only the middle range of the MIDI notes (from 24 to 95) is used.

\section{EVALUATION}

An experiment was designed to verify the strengths and weaknesses of the multimodal data visualization system over traditional tactile diagrams. The comparison study was conducted on a group of visually impaired and blind people. The experiment was run under two conditions, within subjects and counter-balanced. The hypothesis was that the multimodal data visualization system would provide better information comprehension to users than the conventional tactile diagrams.

\section{Experiment Setup}

There were two conditions in the experiment: the tactile diagram condition and the multimodal system condition. Bar chart representation was used as the experimental platform and the data used to construct the bar charts were taken from the website of the UK Department of Health [11]. The data showed the statistics regarding the ward attendance, bed availability and out patient rates of hospitals in England from 1993 to 2000.

The tactile diagrams used in the experiment are designed and raised by the National Tactile Diagram Centre [12]. A sample tactile diagram is shown in Figure 4. There is a small gap $(3 \mathrm{~mm})$ between each bar.

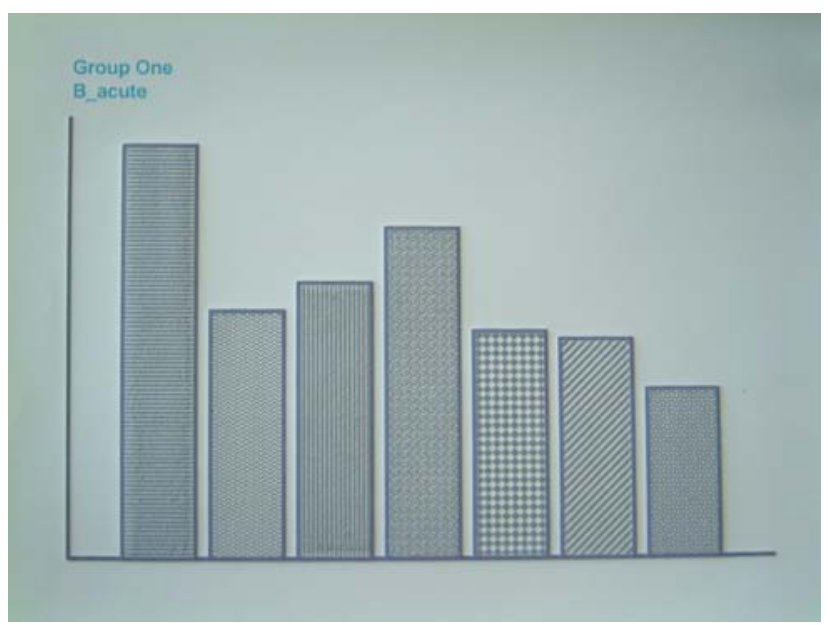

Figure 4. A sample of tactile diagram used in the experiment.

The bar charts used in the multimodal system condition had all the features described in the previous section. Participants could interact with the bar charts using both haptic and audio feedback. In the training section, each feature was explained to the participants in detail. Therefore they knew how to use all the features to extract information from the graph. In the experiment, it was up to them to decide which method of exploration to use.
17 participants were recruited from the Royal Blind College at Hereford. They were students at the college and it was their first time to use the mulitmodal system. Their visual impairment ranged from partially sighted to completely blind. There is a wide range in their age, from 16 to 53 .

Two groups of 10 graphs were used in the experiment. The sequence of which group used first, and under which condition they were used, were all randomized in order to minimize the learning effect and the possible unequal difficulty on the graphs. Participants needed to answer four questions on each graph and had four minutes to do so. The questions were:

Q 1. What is the general trend of the graph?

Q 2. Which bar represents the lowest value?

Q 3. Which bar represents the highest value?

Q 4. Which two bars are the closest in value?

Before the experiment, participants were given a training session in which they would familiarize themselves with the graph features and the experiment procedure. Up to four sample graphs would be given to the participants and the training time was no more than half an hour. These values varied due to the variation between participants' capability. At the end of training, participants would be confident with both media.

The measurements taken in the experiment include the number of correct answers, the time taken to find the answers and the workload index [13] assessed in a questionnaire after the experiment.

\section{Results}

The number of correct answers reflects the accuracy of information extracted from the graphs by the participants. In the tactile diagram condition, the average total number of correct answer was $87.06 \%$ whereas in the multimodal system condition, the figure was $96.03 \%$. The difference between the results is significant (t-test gives $\mathrm{T}_{16}=5.914$, $\mathrm{p}<0.001)$. The numbers of correct answers to the first three questions are very high and similar. This trend changes at the answers to the last question in which data comparison is required. The accuracy drops significantly $\left(\mathrm{T}_{16}=5.734\right.$, $\mathrm{p}<0.001)$ in the tactile diagram condition $(61.76 \%)$. The accuracy in the multimodal system condition for the last question is $85.88 \%$. A diagram showing the results of correct answers is given in Figure 5.

In general, participants used much less time in the tactile diagram condition than in the multimodal condition. The time taken in these two conditions are $24.54 \%$ and $38.21 \%$ of the maximum allowed time respectively. The difference between these two conditions is significant $\left(\mathrm{T}_{16}=7.398\right.$, $\mathrm{p}<0.001)$. 


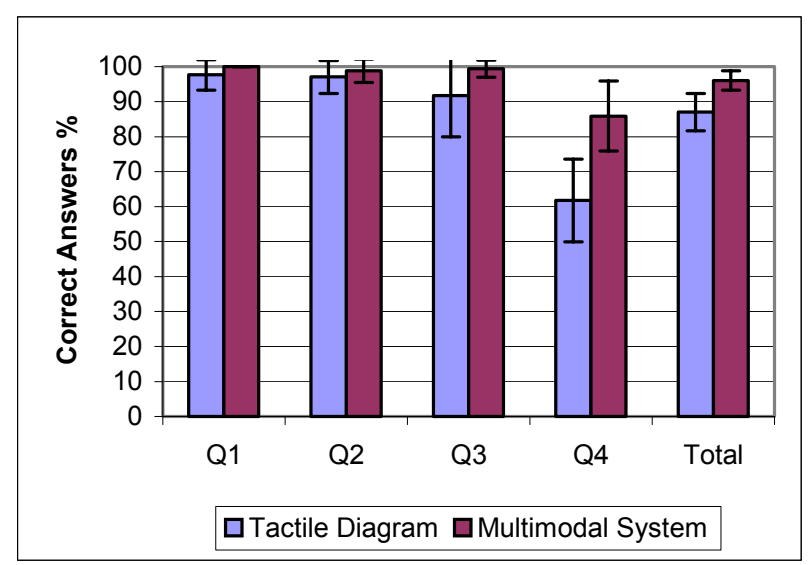

Figure 5. Distributions of participants' number of correct answers.

The workload index consists of six contributing factors: mental, physical \& temporal demands, effort, performance and frustration level [13]. The distributions of participants' ratings on these six factors and the overall workload index are shown in Figure 6. Participants gave higher ratings to the factors in the multimodal system condition and as a result the average overall workload index is $58.84 \%$ which is significantly higher than the one in the tactile diagram condition $(39.86 \%)$. The t-test results shows $\mathrm{T}_{16}=3.742$ and $\mathrm{p}=0.0018$.

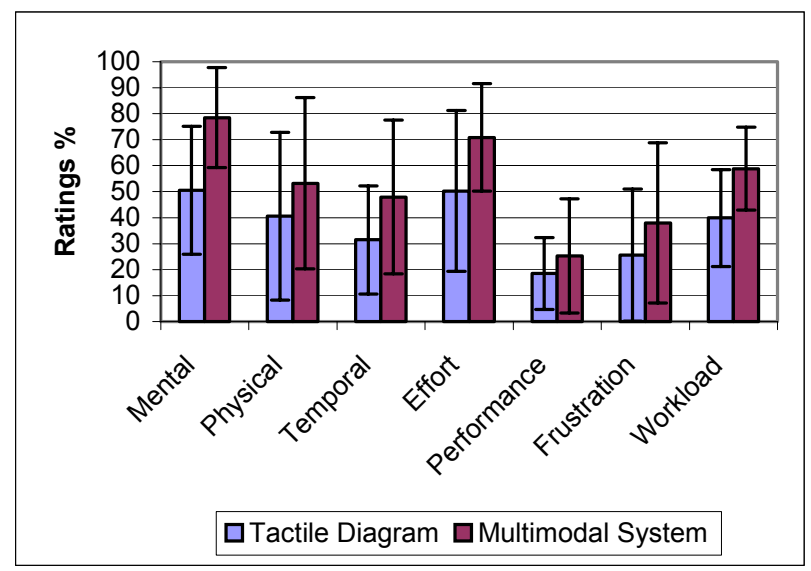

Figure 6. Distribution of participants' ratings in workload assessment.

\section{Discussion}

The main focus of this experiment is to evaluate whether the multimodal system can actually outperform traditional tactile diagrams in presenting graphical information to visually impaired people. The answer to this question relies on the measurements taken in the experiment. They are the number of correct answers, task completion time, workload index and the observations on participants' way of exploration. The results of the experiment have partially supported the hypothesis as the number of correct answers produced by the participants is significantly higher in the multimodal system condition. This means that participants could obtain more accurate information through the VR medium. On the other hand, participants used more time and spent greater effort to achieve this level of accuracy. In order to understand the causes and implications of these results, we need to look at each of them separately.

There were two major types of tasks in the experiment questions. They were trend detection and data comparison. The first three questions required trend detection and simple data comparison whereas the last question involved substantial data comparison. The results showed that participants did well in both conditions. Although the results in the mulitmodal system condition are slightly higher, they were not proven to be significant as there is a ceiling effect. When substantial comparison is required, the VR medium was better as the accuracy of participants' answers is much higher. Therefore, the final score in the multimodal system condition is much higher than the tactile diagram condition. This is due to the effect of speech output. Speech provides a precise form of information which can solve the ambiguities in the haptic and nonspeech audio representation. Therefore, most participants used speech either to find their answers or confirm the answers which had been located. However, it takes time for the information to be read out and thus the time needed for answering the questions is longer.

The long task completion time is also affected by the limitation of the force feedback device used which only provides one single point of contact. The information which can be convey through this one point of contact is very limited. It would take participants a long time to pick up information about a large area of interest. This inefficiency has thus prolonged the participants' exploration time.

Although non-speech audio is available to minimize the effort that has to be spent on the haptic interface, participants are not familiar to this type of audio representation. Even though they understand the relationship between the pitch and bar height, it is still not their usual way of accessing data. Blind people are more used to screen readers which read out information displayed on the screen. Speech is a more direct method to convey information as no translation is required unlike the haptic and non-speech audio cases. However, it takes time for participants to listen and understand the content of the speech. Moreover, they need to manipulate the received information in their short-term memory in order to tell the height difference between bars. This becomes even more demanding in the last question in which different heights between bars had to be compared in order to find the two closest values. This requires good memory for storing data for later comparison. Therefore, a heavy burden is placed on the participants and this has been reflected on their 
ratings on the mental demand and effort which are much higher than the other contributing factors.

Participants indicated that there was more workload in the multimodal system condition but through their comments after the experiment, they generally thought that the interface is easy to use. They suggested if they had more time to practice, they could perform better. As this is their first time to use the multimodal system, the unfamiliarity definitely has a major effect on their workload assessment. Despite the relatively short training time compared with participants' experience on tactile diagrams, they managed to obtain substantially more correct answers in the multimodal system. This has proved the hypothesis of the evaluation. The VR system has the advantage over the paper-based medium and, provided that users have frequent use of the system, task completion time and workload index can be improved.

Some observations have been made on participants' approach to the graphs. Most participants use haptics as a navigation tool which guides them entering and leaving the bars. Although they can use it to determine the height of the bars, they did not use it in this way. Some people were more musically skilled so that they could use the nonspeech sounds to obtain most of the answers. They only needed to use synthesized speech to confirm or find answers to the last question. On the other hand, some participants used speech throughout the experiment, this complete reliance may show that they are more familiar with screen readers. On the tactile diagrams, most participants used two index fingers to feel the height difference between bars. These are usually the dominant fingers trained to read Braille. A gap between bars is useful for them to differentiate one bar from another but the gap should be small enough to be covered by one finger. In the workload assessment, participants felt they had a better performance in the tactile diagram condition. This again shows that they are more confident in the representation which is more familiar.

\section{CONCLUSIONS}

A multimodal data visualization system has been developed to provide graphical information to visually impaired people via their senses of touch and hearing. An evaluation has been conducted to investigate the effectiveness of this system in comparison with the traditional tactile diagram representation. The experimental results have proven the advantages of using the multimodal data representation over the conventional technique. The introduction of audio feedback had a major benefit to users' exploration on the graph and this can be seen from the higher accuracy of participants' answers. Participants perceived the graphs via their audio channel and speech is the major tool used to acquire precise information. Haptic features gave participants the identity of the virtual objects and the sense of their location on the graph. While the effect of the force feedback device's limitations in narrow bandwidth and low efficiency is kept to a minimum, the advantage of the force feedback device in presenting kinesthetic information is fully utilized. Therefore, the integration of audio and haptics provides a positive contribution to users' performance on virtual graphs.

In conclusion, we recommend that the multimodal VR approach is an effective way of presenting graphical information. The longer task completion time and higher workload indication can be overcome by giving users more training and practice. Paper-based medium will be capable of presenting simple information. However, to present more complex data, multimodal VR approach will be more appropriate. Successful use of the system relies on the proper distribution of functionalities and roles to different modalities, haptics and audio in this case, in rendering data. A well-designed system will make full use of the complementary effect of the multimodality so that strengths of each modality will be amplified and limitations will be alleviated. Based on the experimental findings, haptics is suitable for navigation and guidance type of tasks, whereas speech and non-speech audio can be used to present detailed and abstract information. Further work is needed to improve our system and 3D audio will be incorporated.

\section{ACKNOWLEDGEMENTS}

The authors would like to thank the staff and students at the Royal National College for the Blind at Hereford for their participations in the experiment. This research work is funded by EPSRC Grant GR/M44866, ONCE (Spain) and Virtual Presence Ltd.

\section{REFERENCES}

1. Dimigen G., Scott F., Thackeray F., Pimm M., Roy A. W. N., Career expectations of British visually impaired students who are of school-leaving age, Journal of Visual Impairment and Blindness, 87, 1993, p. 209-210.

2. Edman, P.K., Tactile graphics. 1991: American Foundation for the Blind.

3. Lee S., Wiker S. F., Vanderheiden G. C. Interactive haptic interface: two-dimensional form perception for blind access to computers. in 5th International conference on human computer interaction. 1993.

4. Blenkhorn P., Evans D. G., Using speech and touch to enable blind people to access schematic diagrams. Journal of Network and Computer Applications, 1998. 21: p. 17-29.

5. Ramstein C., Martial O., Dufresne A., Carignan M., Chasse P., Mabilleau P.,, Touching and Hearing GUI's: Design Issues for the PC-Access System. ACM ASSETS 96, 1996: p. 2-9.

6. Colwell C., Petrie H., Kornbrot D., Hardwick A., Furner S., Haptic virtual reality for blind computer users. in Annual ACM Conference on Assistive Technologies. 1998. Marina del Rey, CA, USA: ACM. 
7. Wies E. F., Gardner J. A., Sile O'Modhrain M., Hasser C. J., Bulatov V. L., Web-based Touch Display for Accessible Science Education. Haptic HumanComputer Interaction, 2001. LNCS 2058: p. 52-60.

8. $\mathrm{Yu}$ W., Brewster S. A., Comparing Two Haptic Interfaces for Multimodal Graph Rendering. Accepted by IEEE VR2002, 10th Symposium on Haptic Interfaces for Virtual Environment and Teleoperator Systems (Haptics 2002). (Florida USA), 2002.

9. Pengelly H. L., Investigating the use of force feedback devices in human-computer interaction, Master Dissertation, Department of Computing Science, University of Glasgow. 1998.
10. Challis B. P., Edwards A. D. N., Design Principle for Tactile Interaction. Haptic Human-Computer Interaction. LNCS 2058, 2001: p. 17-24.

11. Department of Health, Hospital Activity Statistics. http://www.doh.gov.uk/hospitalactivity/index.htm.

12. National Centre for Tactile Diagrams, University of Hertfordshire, College Lane, Hatfield, Herts, AL10 9AB, UK.

13.Hart S. G., W.C., Workload assessment and predication, in MANPRINT, an approach to systems integration. 1990, Van Nostrand Reinhold: New York. 